\title{
EQUIDISTANT PERMUTATION ARRAYS: A BOUND
}

\author{
G. H. J. VAN REES and S. A. VANSTONE \\ (Received 25 June 1979; revised 24 July 1980, 14 October 1981) \\ Communicated by W. D. Wallis
}

\begin{abstract}
An equidistant permutation array is a $v \times r$ array $A(r, \lambda ; v)$ defined on a $r$-set $X$ such that every row of $A$ is a permutation of $X$ and any two distinct rows agree in precisely $\lambda$ common columns. Define

$$
R(r, \lambda)=\max \{v: \text { there exists an } A(r, \lambda ; v)\} .
$$

In this paper, we show that

$$
R(r, \lambda) \leqslant \max \left\{R(n+1,1), \frac{1}{2}(n+2)^{2}, n^{2}-5 n+7,2+\left\lfloor\frac{\lambda}{\left\lceil\left\lceil\frac{n}{3}\right\rceil\right.}\right\rfloor\right\}
$$

where $n=r-\lambda$. Certain results pertaining to irreducible equidistant permutation arrays are also established.

1980 Mathematics subject classification (Amer. Math. Soc.): 05 B 15.
\end{abstract}

\section{Introduction}

An equidistant permutation array "EPA" $A(r, \lambda ; v)$ is a $v \times r$ array defined on a set $X$ of $r$ symbols such that every row is a permutation of $X$ and any two distinct rows have precisely $\lambda$ common column entries. Define $R(r, \lambda)$ to be the maximum value of $v$ such that an $A(r, \lambda ; v)$ exists. A number of results on $R(r, \lambda)$ have been obtained. In particular, the asymptotic behaviour of this function was shown in [11].

$$
R(r, \lambda)=2+\left\lfloor\frac{\lambda}{\left\lceil\frac{n}{3}\right\rceil}\right\rfloor \text { whenever } \lambda>\left(\left\lceil\frac{n}{3}\right\rceil\right)\left(n^{2}+n\right)
$$

(c) Copyright Australian Mathematical Society 1982 
where $n=r-\lambda$ and $\lfloor x\rfloor$ and $\lceil x\rceil$ are respectively, the greatest integer less than or equal to $x$ and the least integer greater than or equal to $x$. In [6], it is shown that

$$
R(r, \lambda) \leqslant \max \left\{R(n+1,1), \frac{1}{2}(n+2)^{2}, n^{2}-2 n+4,2+\left\lfloor\frac{\lambda}{\left.\left[\frac{n}{3}\right\rceil\right]}\right\} .\right.
$$

One of the results of this paper is to improve this bound. As for a lower bound, it follows immediately from the results in [4] that

$$
R(r, \lambda) \geqslant 2 n-4 \text { whenever } n \geqslant 5 \text {. }
$$

There are a number of constructions for EPAs which exceed this bound but there is no known uniform lower bound which is better than this one.

An EPA is said to be reducible if it contains at least one column $c_{j}$ such that every entry in this column is identical. An EPA which is not reducible is irreducible. Define $R_{1}(r, \lambda)$ to be the maximum value of $v$ such that there exists an irreducible $A(r, \lambda ; v)$. In [5] it is shown that

$$
R_{1}(r, \lambda) \geqslant(\lambda+1)(n-2)+1
$$

if there exists a set of $\lambda+1$ mutually orthogonal latin squares of order $n-2$ with two disjoint common transversals. Section 3 establishes that if $1<\lambda \leqslant n$, then

$$
R_{1}(r, \lambda) \leqslant \max \left\{\frac{(n+2)^{2}}{2},(\lambda+1)(n-2)+1\right\} .
$$

We also evaluate $R_{1}(r, \lambda)$ exactly for an infinite number of values of $r$ and $\lambda$.

\section{Preliminaries}

Rather than study EPAs directly, consider the closely related system called orthogonally resolvable $(r, \lambda)$-designs. To do this, we require a number of definitions.

An $(r, \lambda)$-design $D$ is a collection $B$ of subsets (called blocks) of a finite set $V$ of elements (called varieties) such that any two distinct varieties of $V$ are contained in precisely $\lambda$ common blocks and every variety is contained in exactly $r$ blocks of $D$. In this paper $n$ will always equal $r-\lambda$. A block is said to be complete if it contains all the varieties of $D$. A block of cardinality (or size) one is called a singleton (block).

It is important to distinguish the following two special classes of $(r, \lambda)$-designs. An $(r, \lambda)$-design which only has blocks of size $1, v-1$ or $v$, where $v=|V|$, is called near-trivial. 
An $(r, \lambda)$-design $D$ is said to be resolvable or contain a resolution $R$ if the blocks of $D$ can be partitioned into classes (called resolution classes) such that every variety of $D$ is contained in precisely one block of each resolution class. We say that an $(r, \lambda)$-design $D$ is orthogonal or orthogonally resolvable (denoted by $O D(r, \lambda)$ ) if $D$ contains resolutions $R$ and $R^{\prime}$ and $R_{1}, R_{2}, \ldots, R_{r}$ and $R_{1}^{\prime}$, $R_{2}^{\prime}, \ldots, R_{r}^{\prime}$ are the resolution classes of $R$ and $R^{\prime}$ respectively such that for all $i$ and $j(1 \leqslant i, j \leqslant r) R_{i}$ and $R_{j}^{\prime}$ have at most one labelled block in common. The blocks of $D$ are considered labelled so that a given subset can occur repeatedly as distinct blocks.

The connection between equidistant permutation arrays and orthogonal designs is given in the following theorem proved by Deza, Mullin and Vanstone [2].

THEOREM 2.1. An $A(r, \lambda ; v)$ exists if and only if an $O D(r, \lambda)$ with $v$ varieties exists.

An orthogonal design will be defined in this paper to be irreducible if it does not contain a complete block. Then noting that a complete block in an orthogonal design corresponds to a column containing only one symbol in the corresponding EPA, Theorem 2.1 can be extended to the following theorem.

THEOREM 2.2. An irreducible $A(r, \lambda ; v)$ exists if and only if an irreducible $O D(r, \lambda)$ with $v$ varieties exists.

We will use the concept of orthogonal designs to establish results on EPAs. Before we are able to do this several more definitions and known results on $(r, \lambda)$-designs are required.

Let $D$ be an $(r, \lambda)$-design on $v$ varieties and let $x$ be a variety in $D$ which occurs in blocks $B_{1}, B_{2}, \ldots, B_{r}$. If $V$ denotes the variety set of $D$, then the $r$-complement, $\bar{D}_{x}$, of $D$ with respect to $x$ is the system obtained from $D$ by replacing $B_{i}$ $(1 \leqslant i \leqslant r)$ to $V \backslash B_{i}$. It is easily verified that $\bar{D}_{x}$ is a $(2 n, n)$-design on $v-1$ varieties. If $\lambda \leqslant n$, the augmented $r$-complement, $\bar{D}_{x}^{*}$, of $D$ with respect to $x$ is the system obtained from $D$ by replacing $B_{i}(i=1,2, \ldots, n+\lambda)$ by $V \backslash\left(B_{i} \backslash\{x\}\right)$ and then adjoining $n-\lambda$ blocks consisting of $\{x\}$.

The next result due to Deza [1], puts some restrictions on the block sizes in $(r, \lambda)$-designs.

THEOREM 2.3. If $D$ is an $(n+\lambda)$-design having $v$ varieties and block sizes $k_{1}$, $k_{2}, \ldots, k_{i}$, then $k_{i}\left(v+1-k_{i}\right) \leqslant n(v+1), 1 \leqslant i \leqslant t$. 
If $v>\frac{1}{2}(n+2)^{2}$, then Theorem 2.1 can be used to show that all the blocks in $D$ have size either at least $\frac{1}{2}\left(n^{2}+2 n+2\right)$ (called Big blocks) or at most $n+1$ (called Little blocks). Also the Big blocks contain over half the varieties.

The following two theorems will be used extensively in the sequel. The first theorem was proved by Hall [3] and in a slightly weaker version by Vanstone and McCarthy [11]. The second theorem was proved by Vanstone [9]. Because the proofs are lengthy, they will not be given here.

THeOREM 2.4. Let $D$ be $a(2 n, n)$-design on $v \geqslant \frac{1}{2}(n+2)^{2}$ varieties. Then precisely one of the following is true.

(i) $D$ contains $n-l$ Big blocks all of which are complete.

(ii) $D$ contains $n$ complete blocks and $D$ is trivial.

(iii) $D$ contains $n+1$ Big blocks and every variety of $D$ is contained in exactly $n$ of these blocks.

(iv) $D$ contains $n+1$ Big blocks. One variety of $D$ occurs in all the Big blocks and every other variety occurs in exactly $n$ Big blocks.

TheOREM 2.5. Let $D$ be an $(r, \lambda)$-design having $v>\frac{1}{2}(n+2)^{2}$ varieties and $l$ Big blocks, $B_{1}, B_{2}, \ldots, B_{1}$. Assume also that $D$ is not near-trivial. If $B_{i}$ is replaced by $V \backslash B_{i}$ for all $i, 1 \leqslant i \leqslant l$, then either

(a) the resulting blocks form an $(n+1,1)$-design on the variety set $V$ or

(b) some variety $x \in V$ only occurs in $n-1$ blocks of size one and the remaining blocks form an $(n+1,1)$-design on the variety set $V \backslash\{x\}$.

The resultant $(n+1,1)$-design will be denoted by $\widehat{D}_{B}$.

\section{Upper bounds for $R_{1}(r, \lambda)$}

We will use the results of the previous section to improve the upper bounds on the number of varieties in an irreducible orthogonal non-near-trivial design (IONN designs) with $v>\frac{1}{2}(n+2)^{2}$ and $\lambda>1$.

LEMMA 3.1. In an $O D(n+\lambda, \lambda)$ with $v>\frac{1}{2}(n+2)^{2}$, if a resolution class contains a Big block which is not complete, then that resolution class contains only that Big block and singletons.

Proof. Assume that a resolution class contains a Big block $B_{1}$, and a block $B_{2}$ of size greater than one. Since Big blocks contain more than half the varieties of $D, B_{2}$ is not a Big block. Now, $B_{2}$ contains a pair of varieties, $a$ and $b$, neither of 
which is contained in $B_{1}$. Then, by Theorem 2.5 ., if $\bar{D}_{B}$ is formed, an $(n+1,1)$ design on $v$ or $v-1$ varieties will result. In this design, $\vec{B}_{1}$, the complement of $B_{1}$, and $B_{2}$ both contain $a$ and $b$. But this contradicts $\lambda=1$ and if $x$ is a singleton block in that resolution class containing the Big block $B_{1}$, then in the orthogonal resolution, $x$ occurs in a different singleton block.

LEMMA 3.2. In an IONN $(n+\lambda, \lambda)$-design $(\lambda>1)$ having $v>\frac{1}{2}(n+2)^{2}$ varieties, the number of Big blocks is $\lambda+2 k+1$ and every variety except possibly one occurs in $\lambda+k$ Big blocks for some $k \geqslant 0$.

Proof. Assume a variety, $x$, is in $\lambda-2$ or fewer Big blocks of $D$. Thus, $x$ is in $n+2$ or more little blocks of $D$. Then $\bar{D}_{x}$ is a $(2 n, n)$-design with at least $n+2$ Big blocks. But this contradicts Theorem 2.4.

So let us assume $x$ is in exactly $\lambda-1$ Big blocks. Thus, it is in $n+1$ Little blocks of $D$. Then, $\bar{D}_{x}$ is in $(2 n, n)$-design with at least $n+1$ Big blocks. By Theorem 2.4, this is the maximum number of Big blocks possible in $\bar{D}_{x}$. So, in $D$, there are only these $\lambda-1$ Big blocks. But these blocks would have to be complete blocks which is a contradiction of $D$ being irreducible. Thus every variety occurs in at least $\lambda$ Big blocks and, since complete blocks are ruled out, there must be at least $\lambda+1$ Big blocks.

Let there be $\lambda+p$ Big blocks in $D$. Since $p>0$, there can only be one variety, say $\infty$, that occurs in every Big block. Since every variety occurs in at least $\lambda$ Big blocks, $\infty$ does not occur with any variety in a Little block.

Since $D$ is non-near-trivial either a Little block, $B_{1}$, of size greater than or equal to two must exist or a Big block, $B_{2}$, of size less than $v-1$ must exist. If $B_{1}$ exists, let $x$ be a variety of $B_{1}$. If $B_{1}$ does not exist, let $x$ be a variety of the complement of $B_{2}$. Then $x$ occurs in $\lambda+t$ Big blocks where $t<p$ and $t \geqslant 0$. Thus $p-t$ Big blocks do not contain $x$. Also $x$ is contained in $n-t$ Little blocks. Therefore $\bar{D}_{x}$ is a $(2 n, n)$-design which contains $(p-t)+(n-t)=p+n-2 t$ Big blocks, all of which cannot be complete due to either $B_{2}$ or the complement of $B_{1}$. Then Theorem 2.3 states that $\bar{D}_{x}$ is a $(2 n, n)$-design with $n+1$ Big blocks. Hence $p+n-2 t=n+1$ or $2 t=p-1$. Let $p=2 k+1$, then $t=k$.

If any other variety $y \neq \infty$ is $r$-complemented the number of Big blocks is either $n+1, n$ or $n-1$. It is not $n$ as $p+n-2 t=n$ implies $p$ is even. Thus every other variety occurs in either $\lambda+k$ or $\lambda+k+1$ Big blocks. If $y$ occurs in $\lambda+k+1$ Big blocks, it must occur in $\lambda$ Big blocks with $x$ and every Big block must contain an $x$ or a $y$ or both. But then in $\bar{D}_{B}$, no block contains the pair $x y$ which contradicts Theorem 2.5 as $x, y \neq \infty$.

If $k>0$, then all varieties occur in $\lambda+k$ Big blocks. If $k=0$, $\infty$ may occur in each Big block and every other variety occurs in $\lambda$ Big blocks. Note the similarity to Theorem 2.4 . 
THEOREM 3.3. If $1<\lambda \leqslant n$ then

$$
R_{1}(r, \lambda) \leqslant \max \left\{\frac{1}{2}(n+2)^{2},(\lambda+1)(n-2)+1\right\} .
$$

Proof. Let $D$ be an IONN $(n+\lambda, \lambda)$-design having $v>\frac{1}{2}(n+2)^{2}$. The blocks of $D$ can be partitioned into Big and Little blocks. Suppose some variety $x$ is missing from at least two Big blocks of $D$. The augmented $r$-complement of $x$ is a $(2 n, n)$-design with at least one element missing from at least two Big blocks. This contradicts Theorem 2.4. Thus every variety of $D$ is missing from at most one Big block. By Lemma 3.2, this implies that $D$ contains precisely $\lambda+1$ Big blocks and every variety, except possibly the variety $\infty$, is contained in exactly $\lambda$ of these blocks.

Let $B_{1}, B_{2}, \ldots, B_{\lambda+1}$ be the Big blocks. Since every variety of $D$ except $\infty$ is in $\lambda$ Big blocks, it follows that $\bar{B}_{i} \cap \bar{B}_{j}=\varnothing 1 \leqslant i<j \leqslant \lambda+1$ and that $\cup_{i=1}^{\lambda+1} \bar{B}_{i}=$ $V \backslash\{\infty\}$ where $V$ is the variety set of $D$. Let $x \in B_{j}$ and $x$ be different from $\infty$. Now $x$ must occur with each of the varieties of $B_{j}$ in Little blocks of $D$. The elements of $\bar{B}_{B}$ would have a pair of varieties occurring twice which is a contradiction in an $(n+1,1)$-design. Hence $x$ must occur in at least $n-1$ of these Little blocks. Lemma 3.1 implies that $x$ is contained in at least two singleton blocks as $x$ is missing from one Big block. Hence $x$ is contained in at least $n-1+2+1=n+2$ blocks of $\bar{D}_{B}$ which contradicts the fact that it is an $(n+1,1)$-design. Therefore $\left|\bar{B}_{j}\right| \leqslant n-2$, for all $j, 1 \leqslant j \leqslant \lambda+1$ and $|V| \leqslant$ $(\lambda+1)(n-2)+1$.

If, on the other hand, $D$ is an irreducible, orthogonal near-trivial $(n+\lambda, \lambda)$ design then it can have at most $n+\lambda-1$ varieties which is less than $\frac{1}{2}(n+2)^{2}$ for $\lambda \leqslant n$.

COROLLARY 3.4. If $n-2$ is a prime power greater than 16 and $\frac{1}{2}(n+4)<\lambda \leqslant$ $n-5$ is a positive integer then $R_{1}(r, \lambda)=(\lambda+1)(n-2)+1$.

Proof. When $n-2$ is a prime power, the construction of [5] gives an $O D(r, \lambda)$ having $(\lambda+1)(n-2)+1$ varieties. This and the bound in the preceding theorem give the desired result.

We now prove a partial converse to theorem given in [5].

THEOREM 3.5. Let $n, \lambda, \alpha$ be positive integers such that $\frac{1}{2}(n+4)<\lambda \leqslant n-5$, $n-2>\frac{1}{2}\left(\alpha^{4}+2 \alpha^{3}+2 \alpha^{2}+3 \alpha\right)$ where $\alpha=n-\lambda-3$. Then $R_{1}(r, \lambda)=$ $(\lambda+1)(n-2)+1$ if and only if there exists a set of $\lambda+1$ pairwise orthogonal Latin squares of order $n-2$ having two disjoint common transversals. 
Proof. The sufficiency has been shown by the construction in [5].

Suppose $R_{1}(r, \lambda)=(\lambda+1)(n-2)+1$. Then $v \geqslant \frac{1}{2}(n+8)(n-2)+1>$ $\frac{1}{2}(n+2)^{2}+1$ for appropriate $n$. Then as in the previous theorem we may assume that $D$ is an IONN $(n+\lambda, \lambda)$-design having $\lambda+1$ Big blocks. Also the complements of the Big blocks are disjoint and of maximum size $n-2$. Since $v=$ $(\lambda+1)(n-2)+1$, each complement must be of size $n-2$ and there must be an $\infty$ variety in $D$. Since a variety (except for $\infty$ ) is missing from exactly one Big block, it occurs twice as a singleton by Lemmata 3.1 and 3.2. Furthermore no singletons can occur in the intersection of two resolution classes containing Big blocks. Then in $\bar{D}_{B}$, if two singletons are deleted for every other variety, an $(n-1,1)$-design is obtained. The blocks for this design are the $\lambda+1$ complements of the Big blocks and the Little blocks which occur in the $(n-1)^{2}$ intersections of the resolution classes which contain no Big blocks. (An intersection need not contain a block.)

Now any $(n+\lambda, \lambda)$-design with $(\lambda+1)(n-2)$ varieties and at most $n^{2}-2 n$ $+\lambda-2$ blocks of which $\lambda+1$ are of size $n-2$ is an example of a pseudoparallel complement and it has been shown by Mullin and Vanstone [8] that whenever $n-2>\frac{1}{2}\left(\alpha^{4}+2 \alpha^{3}+2 \alpha^{2}+3 \alpha\right)$ where $\alpha=n-\lambda-3$ that this design is embeddable in an affine plane of order $n-2$. This implies the existence of a set of $t$ $(1 \leqslant t \leqslant n-4)$ pairwise orthogonal Latin squares of order $n-2$ having two distinct common transversals.

\section{An improved upper bound for $R(r, \lambda)$}

In this section, we obtain a universal bound for $R_{1}(n+\lambda, \lambda)$ by calculating limits on the size of the Little blocks and the number of Big blocks. This leads to an improved bound for $R(r, \lambda)$. These next lemmata will prove useful throughout this section.

LEMMA 4.1. In an IONN design $D$, with $v>\frac{1}{2}(n+2)^{2}$ and $\lambda>1$, any two varieties can occur together in at most one of a Little block or a complement of a Big block.

Proof. Suppose that in $D$ there are two varieties, $a$ and $b$ which occur together either in two Little blocks or in a Little block and the complement of a Big block. If $\bar{D}_{B}$ is formed, then an $(n+1,1)$-design exists with the pair $a b$ occurring twice. This is a contradiction. 
Corollary 4.2. In an IONN design D with $v>\frac{1}{2}(n+2)^{2}, \lambda>1$ and $\lambda+2 k$ +1 Big blocks, if $B_{1}$ is the largest Little block, then the number of Big blocks is greater than or equal to $(k+1)\left|B_{1}\right|$.

Proof. Every variety of $B_{1}$ occurs in $k+1$ complements of Big blocks by Theorem 3.2 and the previous lemma states that these blocks are distinct.

The next lemma is one of the fundamental lemmata in the study of $(r, 1)$-designs and is useful in the later lemmata.

Lemma 4.3. In a non-trivial $(r, 1)$-design, $D$, the size of a block is less than or equal to $r$.

Proof. Assume a block, $B_{1}$, in $D$ has size at least $r+1$. Since $D$ is non-trivial, there is a variety $x \notin B_{1}$ and $x$ must occur with the varieties of $B_{1}$ in distinct blocks as $\lambda=1$. Hence $x$ occurs at least $r+1$ times which is a contradiction.

With this lemma we can now limit the size of the blocks in an $(n+\lambda, \lambda)$ design.

LEMMA 4.4. In an IONN $(n+\lambda, \lambda)$-design with $v>\frac{1}{2}(n+2)^{2}, \lambda+2 k+1$ Big blocks and $\lambda>1$, the size of a Little block and the complement of a Big block is at most $n-k$.

Proof. By Lemma 3.2, a variety, $x$, occurs in $\lambda+k$ Big blocks which means it does not occur in $k+1$ Big blocks. These Big blocks are all in distinct resolution classes and by Lemma 3.1, $x$ occurs as a singleton in these resolution classes. This is true for every variety except $\infty$. Then in $\bar{D}_{B}$, if $k+1$ singletons are deleted for every variety, an $(n-k, 1)$-design is obtained. This design has block size at most $n-k$ by Lemma 4.3 .

Thus a variety in the above $(n-k, 1)$-design occurs in $n-k$ blocks of size at most $n-k$. This implies there are at most $1+(n-k)(n-k-1)$ varieties in this design and in the original design $D$ (not counting $\infty$ ). Thus we have proved the following theorem.

THEOREM 4.5. In an IONN $(n+\lambda, \lambda)$-design $D$, with $v>\frac{1}{2}(n+2)^{2}$ and $\lambda>1$, if the number of Big blocks is $\lambda+5$ or more then $v \leqslant n^{2}-5 n+7$.

Next we consider the case in which the design has $\lambda+3$ Big blocks. 
THEOREM 4.6. In an IONN $(n+\lambda, \lambda)$-design $D$, with $v>\frac{1}{2}(n+2)^{2}$ and $\lambda>1$, if the number of Big blocks is $\lambda+3$ then $v \leqslant n^{2}-5 n+7$.

Proof. Let the design $D$ have orthogonal resolutions $R$ and $R^{\prime}$ where $R_{1}$, $R_{2}, \ldots, R_{n-3}$ and $R_{1}^{\prime}, R_{2}^{\prime}, \ldots, R_{n-3}^{\prime}$ are the respective resolution classes which do not contain Big blocks and $R_{n-2}, R_{n-1}, \ldots, R_{n+\lambda}$ and $R_{n-2}^{\prime}, R_{n-1}^{\prime}, \ldots, R_{n+\lambda}^{\prime}$ are the respective resolution classes which do contain Big blocks. Notice that $D$ contains no variety in all Big blocks.

By Lemma 4.4, the size of a Little block is at most $n-1$. Let $B_{1}$ be a Little block that has size $n-1$. Without loss of generality, assume that $B_{1}$ is in resolution classes $R_{1}$ and $R_{1}^{\prime}$. Let $x$ be a variety that occurs in block $B_{2}$ in resolution class $R_{1}$. Now by Theorem 3.2, $x$ does not occur in two Big blocks. By Lemma 3.1, $x$ must occur in singleton blocks in the two resolution classes of $R$ which contain those two Big blocks. Hence in $\bar{D}_{B}, x$ occurs in a singleton block twice, in $B_{2}$, and in $n-1$ distinct blocks containing varieties of $B_{1}$. This implies that $x$ occurs $n+2$ times in an $(n+1,1)$-design which is a contradiction. Therefore Little blocks have size at most $n-2$.

Let $\bar{B}_{3}$ be the complement of a Big block $B_{3}$. Assume that $\bar{B}_{3}$ has size $n-1$. Also assume that the number of Big blocks is greater than $n$. By Theorem 3.2, each variety of $\bar{B}_{3}$ occurs in one more complement of a Big block. Hence the varieties of $\bar{B}_{3}$ occur in $n$ complements of Big blocks. Let $x$ be a variety that occurs in a complement of a Big block $\bar{B}_{4}$ that does not contain any varieties of $\bar{B}_{3}$. Now by Lemma $3.1, x$ must occur in a singleton block in the two resolutions which contain the Big blocks not containing $x$. Hence in $\bar{D}_{B}, x$ occurs in two singleton blocks, in $\bar{B}_{4}$ and in $n-1$ distinct blocks containing varieties of $\bar{B}_{3}$. Again this is a contradiction.

So let us assume that there are at most $n$ Big blocks. Therefore the number of varieties (including multiplicities) in the complements of the Big blocks is at most $n(n-1)$. But by Theorem 3.2 each variety occurs twice in these blocks so that the number of varieties in $D$ is $\frac{1}{2} n(n-1)$. But $\frac{1}{2} n(n-1) \leqslant \frac{1}{2}(n+2)^{2}$ which is a contradiction. Therefore the complement of a Big block has size at most $n-2$.

Consider resolution class $R_{1}$. If there are no blocks of $R_{1}$ which are also in resolution class $R_{1}$ for $i>n-3$ then any variety of $D$ must occur in one of the $n-3$ Little blocks of $R_{1}$ of size at most $n-2$. Hence, there are at most $(n-3)(n-2)$ varieties. If there is such a block it is a singleton block by Lemma 3.1. Let $x$ be the singleton. By Lemma 3.1, $x$ occurs in a singleton block in the two resolution classes of $R$ which contain Big blocks which do not contain $x$. Hence in $\bar{D}_{B}, x$ occurs in three singleton blocks and in $n-2$ blocks of size at most $n-2$. This implies that there are at most $1+(n-2)(n-3)=n^{2}-5 n+7$ varieties in $\bar{D}_{B}$ and in $D$. 
Finally we consider the case when the design has $\lambda+1$ Big blocks.

THEOREM 4.7. In an IONN $(n+\lambda, \lambda)$-design, $D$, with $v>\frac{1}{2}(n+2)^{2}, n \geqslant 5$ and $\lambda>1$, if there are $\lambda+1$ Big blocks then $v \leqslant n^{2}-5 n+7$.

Proof. The size of a Little block or the complement of a Big block is at most $n$ by Lemma 4.4. As in the previous theorem, $D$ has orthogonal resolutions $R$ and $R^{\prime}$ where the first $n-1$ resolution classes do not contain Big blocks while the remaining resolution classes do.

Let $B_{n}$ be a Big block occurring in the intersection of resolution classes $R_{n}$ and $R_{n}^{\prime}$. Let us assume that $\left|\bar{B}_{n}\right|=n$ or $n-1$. Then let $x$ be a variety of any other complement of a Big block. By Lemmata 3.1 and 3.2 every variety of $D$ occurs in at least two singleton blocks. So in $\bar{D}_{B}, x$ occurs in three blocks which do not contain varieties of $\bar{B}_{n}$ and in $n$ or $n-1$ blocks which do. This contradicts $\bar{D}_{B}$ being an $(n+1,1)$-design. So the size of the complement of a Big block is at most $n-2$.

Let the size of a Little block, $B_{1}$, be $n$ or $n-1$. Let $B_{1}$ be the intersection of resolution classes $R_{1}$ and $R_{1}^{\prime}$. Consider a variety $y$ which occurs in a block which is the intersection of resolution class $R_{1}$ and a resolution class $R_{1}^{\prime}, i \neq 1$, which does not contain a Big block. Since $y$ must occur as a singleton twice, $y$, in $\bar{D}_{B}$, occurs in three blocks no containing varieties of $B_{1}$ and $n$ or $n-1$ blocks which do. This contradicts $\bar{D}_{B}$ being an $(n+1,1)$-design.

Let $B_{1}$ and $\bar{B}_{n}$ be as before except $B_{1}$ has size $n-2$ and $\vec{B}_{n}$ contains no varieties of $B_{1}$. Let $x$ be a variety of $\bar{B}_{n}$. Then the singleton blocks containing $x$ must occur in the intersection of resolution classes $R_{1}$ and $R_{1}^{\prime}$ and resolution classes $R_{1}^{\prime}$ and $R_{n}$. If not, $x$ occurs in a Little block of $R_{1}$ (or/and $R_{1}^{\prime}$ ) containing no variety of $B_{1}$. Then in $\bar{D}_{B}, x$ would be in four blocks which contain no varieties of $B_{1}$ and $n-2$ blocks which do. This is a contradiction. Since this argument is true for any variety in $\bar{B}_{n}, \bar{B}_{n}$ must be a singleton.

Now assume a Little block $B_{2} \neq B_{1}$ has size $n-2$ and does not contain $x$. Let $B_{2}$ be in the intersection of resolution class $R_{i}$ and $R_{j}^{\prime}$. As in the previous paragraph $x$ must occur as a singleton in the intersection of $R_{i}$ and $R_{n}^{\prime}$ and $R_{j}^{\prime}$ and $R_{n}$. But this implies $i=j=1$ and $B_{2}=B_{1}$. So the only Little blocks of size $n-2$ are $B_{1}$ and possibly blocks containing $x$.

Let us further assume that there exists another complement, $\bar{B}_{n+1}$, of a Big block which contains no varieties from $B_{1}$. Then the previous arguments must hold for it as well as $\bar{B}_{n}$ so $\bar{B}_{n+1}$ is a block containing one element, say $y$. And the only Little blocks of size $n-2$ are $B_{1}$ and possibly blocks containing $y$. Combining this with the previous paragraph proves that the only Little blocks of size $n-2$ are $B_{1}$ and possibly the block containing the pair $x y$. Thus $x$, in $\bar{D}_{B}$, 
occurs in three singleton blocks, one block of size at most $n-2$ and $n-3$ blocks of size at most $n-3$. Hence the number of varieties in $\bar{D}_{B}$ is at most $1+n-3$ $+(n-3)(n-4)=n^{2}-6 n+10$ and in $D$, at most $n^{2}-6 n+11<n^{2}-5 n+$ 7 for $n \geqslant 5$.

Thus there is at most one complement, $\bar{B}_{n}$, of a Big block which contains no varieties of $B_{1}$. This implies that the $\lambda+1 \leqslant n-2+1$.

Now consider a variety which is not in $B_{1}$, say $z$, which occurs in a complement, $\bar{B}_{n+1}$, of a Big block. Let $\bar{B}_{n+1}$ occur in the intersection of resolution classes $R_{n+1}$ and $R_{n+1}^{\prime}$. Now $z$ must occur as a singleton twice, but assume that no singleton occurs in the intersection of $R_{1}$ and $R_{n+1}^{\prime}$ and $R_{1}^{\prime}$ and $R_{n+1}$. Then $z$ must occur in resolution classes $R_{1}$ and $R_{1}^{\prime}$ in blocks that contain no varieties of $B_{1}$. Then, in $\bar{D}_{B}, z$ must occur in 4 blocks which contain no variety of $B_{1}$ and $n-2$ blocks which do. Again this is a contradiction. Hence any element $z$ which is not in $B_{1}$ must occur as a singleton either in the intersection of $R_{1}$ and $R_{n}^{\prime}$ or the intersection $R_{1}^{\prime}$ and $R_{n}$. But in resolution class $R_{1}$ there is room for only $\lambda+1 \geqslant n-1$ such singletons. Similarly for $R_{1}^{\prime}$. Hence the number of varieties in $\bar{D}_{B}$ is at most $\lambda+1+\lambda+1+\left|B_{1}\right|=3 n$ and the number of varieties in $D$ is at most $3 n+1<\frac{1}{2}(n+2)^{2}$.

We can now assume that the size of a Little block is at most $n-3$ and the size of a complement of a Big block is at most $n-2$. Then since each variety occurs in two singleton blocks and one complement of a Big block, the number of varieties including $\infty$ in $D$ is $2+n-3+(n-2)(n-4)=n^{2}-5 n+7$.

Combining the last three theorems gives the following theorem.

THEOREM 4.8. In an IONN $(n+\lambda, \lambda)$-design with $v>\frac{1}{2}(n+2)^{2}, \lambda>1$, the number of varieties is at most $n^{2}-5 n+7$.

Having this result, the following bound is easily obtained.

THEOREM 4.9.

$$
R(r, \lambda) \leqslant \max \left\{R(n+1,1), \frac{1}{2}(n+2)^{2}, n^{2}-5 n+7,2+\left|\frac{\lambda}{\left[\frac{n}{3}\right\rceil}\right|\right\} .
$$

Proof. Consider an $O D(r, \lambda)$-design $D$ having $v$ varieties. Delete all complete blocks from $D$ to get an $O D\left(r^{\prime}, \lambda^{\prime}\right)$-design $D^{\prime}$ where $r^{\prime}-\lambda^{\prime}=r-\lambda=n$. If $D^{\prime}$ is an IONN design with $\lambda>1$ and with $v>\frac{1}{2}(n+2)^{2}$ we have the bound of Theorem 4.8. If $D^{\prime}$ has $\lambda^{\prime}=1$, the number of varieties is bounded by $R(n+1,1)$. 
Finally, if $D^{\prime}$ is near-trivial, we have the bound

$$
2+\left\lfloor\frac{\lambda}{\left[\frac{n}{3}\right]}\right\rfloor \text {. }
$$

\section{Conclusion}

In order to improve the bound of Theorem 4.9, one must improve the results on $R(n+1,1)$. The best general lower bound for this case as shown in [4] is $R(n+1,1) \geqslant 2 n-4$ whenever $n \geqslant 5$. It was shown in [7] that $R(n+1,1) \leqslant n^{2}$ $-2 n-2$ for $n \geqslant 4$. This is the best upper bound known for this function. It has recently been shown ([13]) that $R\left(q^{2}+q+1,1\right) \geqslant q^{3}+q^{2}$ whenever $q$ is a prime or prime power. This is the first result for $\lambda=1$, showing that $R(r, 1)$ is nonlinear in $r$ for some values of $r$.

\section{Acknowledgement}

The authors wish to thank the referee for many helpful comments.

\section{References}

[1] M. Deza, 'Une propriété extrémale des plans projectifs finis dans une classe de codes equidistants', Discrete Math. 6 (1973), 343-352.

[2] M. Deza, R. C. Mullin and S. A. Vanstone, 'Room squares and equidistant permutation arrays', Ars Combinatoria 2 (1976), 235-244.

[3] J. I. Hall, 'Bounds for equidistant codes and partial projective planes', Discrete Math. 17 (1977), 85-94.

[4] K. Heinrich and G. H. J. van Rees, 'Some constructions for equidistant permutation arrays for index one', Utilitas Math. 13, 193-200.

[5] K. Heinrich, G. H. J. van Rees and W. D. Wallis, 'A general construction for equidistant permutation arrays', Proc. of Conference on Graph Theory and Related Topics, in honour of W.T. Tutte, pp. 247-252.

[6] D. McCarthy and S. A. Vanstone, 'On the maximum number of equidistant permutations', $J$. Stat. Plann. Inference.

[7] R. C. Mullin and E. Nemeth, 'An improved upper bound for equidistant permutation arrays'. Utilitas Math. 13 (1978), 77-85.

[8] R. C. Mullin and S. A. Vanstone, 'On a theorem of Totten', J. Austral. Math. Soc. Ser. A 22 (1976), 494-500.

[9] G. H. J. van Rees, The role of $(r, \lambda)$-designs in some combinatorial configurations, Ph. D. Thesis, Waterloo. 
[10] S. A. Vanstone, 'Irreducible regular pairwise balanced designs', Utilitas Math. 15 (1979), 249-259.

[11] S. A. Vanstone, 'The asymptotic behaviour of equidistant permutation arrays', Canad. J. Math. 31 (1979), 45-48.

[12] S. A. Vanstone and D. McCarthy, '( $r, \lambda)$-designs and finite projective planes', Utilitas Math. 11 (1977), 57-71.

[13] R. Mathon and S. A. Vanstone, 'On the existence of doubly resolvable Kirkman triple systems and equidistant permutation arrays', Discrete Math. 30 (1980), 157-172.

Department of Mathematics

University of Manitoba

Winnipeg, Manitoba R3T 2N2

Canada
Department of Mathematics

St. Jerome's College

University of Waterloo

Waterloo, Ontario N2L 3G1

Canada 